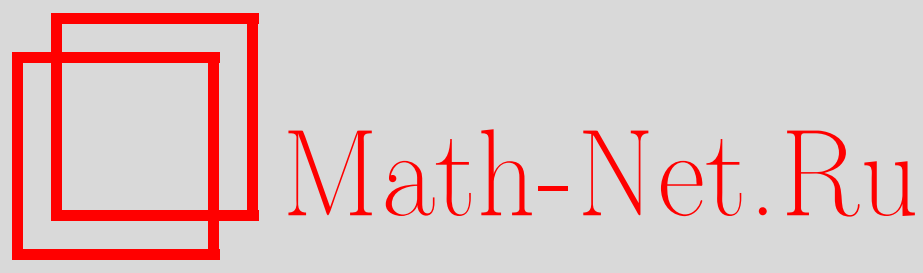

Д. Р. Караханян, Р. Киршнер, Квадратичное разрешение ортогональных и симплектических янгианов, $Т M \Phi$, 2017, том 192, номер 2, 250-258

DOI: https://doi.org/10.4213/tmf9296

Использование Общероссийского математического портала Math-Net.Ru подразумевает, что вы прочитали и согласны с пользовательским соглашением http://www . mathnet.ru/rus/agreement

Параметры загрузки:

IP : 44.207 .124 .84

26 апреля 2023 г., 13:45:29

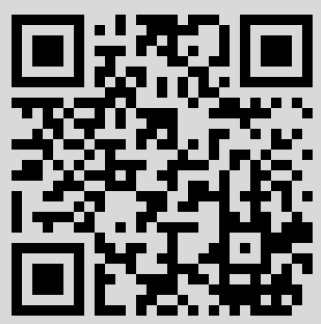




\title{
КВАДРАТИЧНОЕ РАЗРЕШЕНИЕ ОРТОГОНАЛЬНЫХ И СИМПЛЕКТИЧЕСКИХ ЯНГИАНОВ
}

\begin{abstract}
Ортогональные и симплектические янгианы определяются посредством соотношения Янга-Бакстера типа $R L L$, включающего фундаментальную $R$-матрицу, соответствующую $s o(n)$ - или $s p(2 m)$-симметрии. Мы исследовали условия разрешимости второго порядка, при которых разложение $L(u)$ по $u^{-1}$ обрывается после квадратичного члена, и вывели соотношения, соответствующие двум нетривиальным членам разложения $L(u)$.
\end{abstract}

Ключевые слова: интегрируемые системы, соотношение Янга-Бакстера, ортогональные и симплектические алгебры Ли, усеченные янгианы.

DOI: https://doi.org/10.4213/tmf9296

Посвящается памяти П. П. Кулиша

\section{1. ЯНГИАНЫ С ОРТОГОНАЛЬНОЙ И СИМПЛЕКТИЧЕСКОЙ СИММЕТРИЕЙ}

Пусть матрица $R_{12}$ действует в тензорном произведении двух $n$-мерных линейных пространств $V$, ее матричные элементы обозначим как $R_{b_{1} b_{2}}^{a_{1} a_{2}}$. Фундаментальное уравнение Янга-Бакстера имеет вид

$$
\begin{aligned}
R_{b_{1} b_{2}}^{a_{1} a_{2}}(u) R_{c_{1} b_{3}}^{b_{1} a_{3}}(u+v) R_{c_{2} c_{3}}^{b_{2} b_{3}}(v) & =R_{b_{2} b_{3}}^{a_{2} a_{3}}(v) R_{b_{1} c_{3}}^{a_{1} b_{3}}(u+v) R_{c_{1} c_{2}}^{b_{1} b_{2}}(u) \Longrightarrow \\
R_{12}(u) R_{13}(u+v) R_{23}(v) & =R_{23}(v) R_{13}(u+v) R_{12}(u) .
\end{aligned}
$$

Это соотношение широко используется в качестве основы для исследования квантовых интегрируемых систем и рассматривается как аналог тождеств Якоби в формулировке соответствующих алгебр [1]-[7]. Оно имеет решение [7]-[10]

$$
R_{b_{1} b_{2}}^{a_{1} a_{2}}(u)=u(u+\beta) I_{b_{1} b_{2}}^{a_{1} a_{2}}+(u+\beta) P_{b_{1} b_{2}}^{a_{1} a_{2}}-\epsilon u K_{b_{1} b_{2}}^{a_{1} a_{2}},
$$

Работа Р. Киршнера финансировалась программой ОИЯИ (Дубна, Россия) "Гейзенберг-Ландау". Исследования Д. Р. Караханяна поддержаны программой ОИЯИ "Смородинский-Тер-Антонян", а также частично поддержаны Armenian State Committee of Science (грант № SCS 15RF-039).

*Ереванский физический институт, Ереван, Армения. E-mail: karakhan@yerphi.am

${ }^{\dagger}$ Institut für Theoretische Physik, Universität Leipzig, Leipzig, Germany.

E-mail: Roland.Kirschner@itp.uni-leipzig.de 
где

$$
I_{b_{1} b_{2}}^{a_{1} a_{2}}=\delta_{b_{1}}^{a_{1}} \delta_{b_{2}}^{a_{2}}, \quad P_{b_{1} b_{2}}^{a_{1} a_{2}}=\delta_{b_{2}}^{a_{1}} \delta_{b_{1}}^{a_{2}}, \quad K_{b_{1} b_{2}}^{a_{1} a_{2}}=\varepsilon^{a_{1} a_{2}} \varepsilon_{b_{1} b_{2}}, \quad \beta=\frac{n}{2}-\epsilon .
$$

Здесь $\varepsilon_{a b}$ есть невырожденная инвариантная метрика в пространстве $V$ :

$$
\varepsilon_{a b}=\epsilon \varepsilon_{b a}, \quad \varepsilon_{a b} \varepsilon^{b d}=\delta_{a}^{d} .
$$

$R$-матрица (1.2) инвариантна относительно присоединенного действия любой вещественной формы (связанной с метрикой $\left.\varepsilon^{a b}\right)$ комплексных групп $S O(n, \mathbb{C})$ и $S p(n, \mathbb{C})$. Значения $\epsilon=+1$ и $\epsilon=-1$ относятся к группам $S O(n)$ и $S p(n)$ соответственно. Обозначим $\varepsilon^{b d}$ (с помощью верхних индексов) элементы обратной матрицы $\varepsilon^{-1}$.

Существование инвариантного тензора $\varepsilon_{a b}$ приводит к появлению третьего члена в соответствующем выражении для $R$-матриц и к квадратичной зависимости от спектрального параметра $u$.

Пусть $\mathcal{G}$ - алгебра Ли $s o(n)$ или $s p(2 m)(2 m=n)$. Тогда отвечающей ей янгиан $Y(\mathcal{G})$ определяется [5], [6] как ассоциативная алгебра с бесконечным числом генераторов $\left(L^{(k)}\right)_{b}^{a}$, для которых верно условие $\left(L^{(0)}\right)_{b}^{a}=I \delta_{b}^{a}$, где $I$ - единичный элемент в $Y(\mathcal{G})$, и $\left.L^{(k)}\right)_{b}^{a}$ при $k>0$ удовлетворяют определяющим соотношениям. Генераторы $\left.L^{(k)}\right)_{b}^{a} \in Y(\mathcal{G})$ можно считать коэффициентами в разложении оператоpa $L$

$$
L_{b}^{a}(u)=\sum_{k=0}^{\infty} \frac{\left(L^{(k)}\right)_{b}^{a}}{u^{k}}, \quad L^{(0)}=I,
$$

для которого выполняются соотношения Янга-Бакстера типа $R L L$ :

$$
\begin{aligned}
R_{b_{1} b_{2}}^{a_{1} a_{2}}(u-v) L_{c_{1}}^{b_{1}}(u) L_{c_{2}}^{b_{2}}(v) & =L_{b_{2}}^{a_{2}}(v) L_{b_{1}}^{a_{1}}(u) R_{c_{1} c_{2}}^{b_{1} b_{2}}(u-v) \quad \Longleftrightarrow \\
R_{12}(u-v) L_{1}(u) L_{2}(v) & =L_{2}(v) L_{1}(u) R_{12}(u-v),
\end{aligned}
$$

эквивалентные соотношениям, определяющим янгиан $Y(\mathcal{G})$.

Здесь $R_{b_{1} b_{2}}^{a_{1} a_{2}}(u-v)$ есть $R$-матрица Янга-Бакстера (1.2), а во второй строке формулы (1.6) мы использовали стандартные матричные обозначения, принятые в статье [11].

Янгиан $Y(\mathcal{G})$ с бесконечным числом генераторов можно представить с использованием оценок конечного порядка, причем образ генераторов таков, что $\left(L^{(k)}\right)_{b}^{a}=0$ при $k>k_{0}$. В случае общей линейной симметрии уравнение Янга-Бакстера типа $R L L$, включающее фундаментальную $R$-матрицу и оператор $L$, решается с помощью линейного выражения $L_{a b}(u)=u \delta_{a b}+M_{a b}$ без наложения каких-либо ограничений на представления алгебры Ли, порожденные $M_{a b}$, т. е. в универсальной обертывающей алгебре существует решение первого порядка. Ситуация меняется, если рассматривать ортогональные и симплектические симметрии, как в настоящей статье. Ограничение, налагаемое на разложение $L(u)$ по $u$, приводит к условиям связи, которые не могут выполняться одновременно во всех представлениях алгебры Ли. Хорошо известный пример спинорного представления был рассмотрен в статье [12]. Этот и другие примеры были отмечены в работах [9], [13]. Операторы $L$ с простой структурой представляют интерес для приложений, поскольку они являются “строительными блоками" при формулировке квантовых интегрируемых систем. Операторы $L$, соответствующие решениям первого и второго порядков, недавно были рассмотрены в статьях [14], [15]. В настоящей работе мы продолжаем эти исследования, проводя анализ общего решения множества условий разрешимости второго порядка. 


\section{2. УСЛОВИЯ РАЗРЕШИМОСТИ ВТОРОГО ПОРЯДКА}

Рассмотрим квадратичный анзац общего вида (отвечающий случаю $k_{0}=2$ )

$$
L(u)=u^{2}+u G+H
$$

Тогда определяющие соотношения (1.6) записываются как

$$
\begin{aligned}
{\left[u(u+\beta) I_{12}\right.} & \left.+(u+\beta) P_{12}-\epsilon u K_{12}\right]\left((u+v)^{2}+(u+v) G_{1}+H_{1}\right)\left(v^{2}+v G_{2}+H_{2}\right)- \\
& -\left(v^{2}-v G_{2}+H_{2}\right)\left((u+v)^{2}-(u+v) G_{1}+H_{1}\right) \times \\
& \times\left[u(u+\beta) I_{12}+(u+\beta) P_{12}-\epsilon u K_{12}\right]=0 .
\end{aligned}
$$

Это равенство должно выполняться при произвольных значениях спектральных параметров $u$ и $v$, для этого коэффициенты при независимых мономах $u^{k} v^{r}(k+r \leqslant 4)$ должны быть равны нулю. Левую часть (2.2) можно переписать как сумму следующих восьми комбинаций:

$$
\begin{aligned}
& u v^{2}(u+v) \mathfrak{C}^{(2,1)}=\epsilon u v^{2}(u+v)\left[K_{12}, G_{1}+G_{2}\right], \\
& (u+\beta) u v(u+v) \mathfrak{C}^{(2,2)}= \\
& =(u+\beta) u v(u+v)\left(\left[G_{1}, G_{2}\right]+\left(G_{1}-G_{2}\right) P_{12}-\epsilon\left[K_{12}, G_{2}\right]\right), \\
& -u v(u+v) \mathfrak{C}^{(2,3)}= \\
& =-\epsilon u v(u+v) \times \\
& \times\left(K_{12}\left(H_{1}+H_{2}+\left(G_{1}-\beta\right) G_{2}\right)-\left(H_{1}+H_{2}+G_{2}\left(G_{1}-\beta\right)\right) K_{12}\right) \text {, } \\
& -(u+\beta) u(u+v) \mathfrak{C}^{(2,4)}= \\
& =-(u+\beta) u(u+v)\left(\left[G_{1}, H_{2}\right]+\left(H_{1}-H_{2}\right) P_{12}-\epsilon\left[K_{12}, H_{2}\right]\right), \\
& -(u+\beta) v(u+v) \mathfrak{C}^{(2,5)}= \\
& =-(u+\beta) v(u+v)\left(\left[H_{1}, G_{2}\right]+\left(H_{1}-H_{2}\right) P_{12}+\epsilon\left[K_{12}, H_{1}\right]\right), \\
& u v \mathfrak{C}^{(2,6)}= \\
& =-\epsilon u v(u+v) \epsilon u v \times \\
& \times\left(K_{12}\left(H_{1}\left(G_{2}+\beta\right)+\left(G_{1}-\beta\right) H_{2}\right)-\left(H_{2}\left(G_{1}-\beta\right)+\left(G_{2}+\beta\right) H_{1}\right) K_{12}\right), \\
& -u(u+\beta) \mathfrak{C}^{(2,7)}= \\
& =-u(u+\beta)\left(\left[H_{1}, H_{2}\right]+\left(G_{2} H_{1}-H_{2} G_{1}\right) P_{12}-\epsilon K_{12}\left(G_{1}-\beta\right) H_{2}+\right. \\
& \left.+\epsilon H_{2}\left(G_{1}-\beta\right) K_{12}\right) \text {, } \\
& \left.-u \mathfrak{C}^{(2,8)}=-\epsilon u\left(K_{12}\left(H_{1}-\beta G_{1}+\beta^{2}\right) H_{2}-H_{2}\left(H_{1}-\beta G_{1}+\beta^{2}\right)\right) K_{12}\right) \text {. }
\end{aligned}
$$

Данные равенства получены в результате следующих рассуждений: только два члена в (2.2) пропорциональны $u v^{3}$, и после выделения комбинации (2.3) оставшийся пропорциональный $u^{3} v$ член дается формулой (2.4). Отметим, что после выделения (2.3) и (2.4) в соотношении (2.2) остается лишь кубическая зависимость от спектральных параметров; более того, член с $u^{3}$ содержится только в комбинации (2.6), а $u v^{2}$ содержится только в комбинации (2.7). Отделяя эти комбинации, мы можем 
переписать формулу (2.2) в квадратичном по спектральному параметру $u$ виде, который дается суммой комбинаций $(2.8),(2.9)$ и (2.10).

Другими словами, зависимость фундаментальной $R$-матрицы и квадратичных операторов $L$ от спектральных параметров можно преобразовать в указанные выше восемь алгебраических условий (2.3)-(2.10) для операторнозначных матриц $G$ и $H$.

\section{3. РЕШЕНИЕ УРАВНЕНИЙ СВЯЗИ}

Решим теперь упомянутые в предыдущем разделе связи типа $R L L$, используя тождества

$$
P_{12} K_{12}=\epsilon K_{12}=K_{12} P_{12}, \quad K_{12}^{2}=n \epsilon K_{12} .
$$

3.1. Соотношения для случая алгебры Ли. Умножая первое условие (2.3) на $n \epsilon-K_{12}$ слева и справа, получаем, что

$$
\epsilon K_{12}\left(G_{1}+G_{2}\right) K_{12}=n K_{12}\left(G_{1}+G_{2}\right)=n\left(G_{1}+G_{2}\right) K_{12}=2 \operatorname{tr} G K_{12},
$$

поскольку $K_{12} G_{1} K_{12}=K_{12} G_{2} K_{12}=\epsilon \operatorname{tr} G$. Из этого мы можем сделать вывод, что справедливо равенство $G=g+\bar{G}$ :

$$
K_{12}\left(\bar{G}_{1}+\bar{G}_{2}\right)=0=\left(\bar{G}_{1}+\bar{G}_{2}\right) K_{12} \quad \Longrightarrow \quad \operatorname{tr} \bar{G} \cdot K_{12}=0 .
$$

Таким образом, матрица $G$ состоит из бесследовой $\varepsilon$-антисимметричной части $\bar{G}$ и части, имеющей ненулевой след, пропорциональной метрическому тензору. В записи с использованием индексов это означает следующее:

$$
\bar{G}_{a}^{a}=0=\bar{G}_{a}^{a}, \quad \bar{G}_{c_{1} c_{2}}+\epsilon \bar{G}_{c_{2} c_{1}}=0=\bar{G}^{a_{1} a_{2}}+\epsilon \bar{G}^{a_{2} a_{1}} .
$$

Таким образом, мы окончательно приходим к выводу, что первое условие связи для $\mathfrak{C}^{(2,1)}$ в терминах бесследовой матрицы $\bar{G}$ сводится к двум соотношениям

$$
\mathfrak{C}_{L}^{(2,1)}=K_{12}\left(\bar{G}_{1}+\bar{G}_{2}\right)=0, \quad \mathfrak{C}_{R}^{(2,1)}=\left(\bar{G}_{1}+\bar{G}_{2}\right) K_{12}=0 .
$$

Вторая связь

$$
\mathfrak{C}^{(2,2)}=\left[G_{1}+P_{12}-\epsilon K_{12}, G_{2}\right]=\left[G_{1}, G_{2}+P_{12}-\epsilon K_{12}\right]
$$

отражает симметрию матрицы $G$ относительно одновременных поворотов во вспомогательных (первом или втором) и квантовом пространствах. Ее также можно переписать как $\overline{\mathfrak{C}}^{(2,2)}$, используя лишь $\bar{G}$.

Умножая $\overline{\mathfrak{C}}^{(2,2)}$ на $K_{12}$ слева и справа и используя тождества $(3.1)$, получаем равенства

$$
\overline{\mathfrak{C}}_{L}^{(2,2)}=K_{12}\left(\left[\bar{G}_{1}, \bar{G}_{2}\right]-2 \beta \bar{G}_{2}\right), \quad \overline{\mathfrak{C}}_{R}^{(2,2)}=\left(\left[\bar{G}_{1}, \bar{G}_{2}\right]+2 \beta \bar{G}_{2}\right) K_{12} .
$$

Эти выражения также можно рассматривать как условия интегрируемости для третьей связи, поскольку их можно получить, умножая (2.5) на $1-\epsilon P_{12}$ слева и справа соответственно. 
Все полученные до сих пор соотношения касались матрицы $G$ со значениями в алгебре. Она раскладывается на сумму матрицы $g$ с ненулевым следом и градуированной антисимметричной матрицы $\bar{G}$. В этих соотношениях предполагается, что элементы последней являются генераторами алгебры Ли, а $g$ - центральный элемент обертывающей алгебры. Данное предположение верно также и в общем случае, когда разложение не обрывается или обрывается после членов других порядков, отличных от $k_{0}=2$.

Остальные условия связывают второй член $H$ с генераторами $G$ и приводят к ограничениям на множество возможных представлений алгебры Ли.

Рассмотрим более простой пример решения первого порядка [14]. Условия для обрывания разложения следуют из условий, приведенных выше, в которые мы подставляем $H=0$. Первые два выражения не меняются, и единственное новое условие следует из третьего выражения:

$$
\mathfrak{C}^{(2,3)} \longrightarrow \mathfrak{C}^{(1,3)}=K_{12}\left(G_{1}-\beta\right) G_{2}-G_{2}\left(G_{1}-\beta\right) K_{12}=0 .
$$

Оно означает, что бесследовая $\epsilon$-симметричная часть матрицы $\bar{G}^{2}$ исчезает:

$$
\bar{G}^{2}+\beta \bar{G}-\frac{m_{2}}{n}=0, \quad m_{2}=\operatorname{tr}\left(\bar{G}^{2}\right) .
$$

Данное равенство справедливо лишь для некоторых представлений алгебры Ли.

3.2. Часть матрицы $H$, обладающая $\epsilon$-симметрией. Умножая третье выражение $(2.5)$ на $1+\epsilon P_{12}$ слева и справа, мы переписываем его в виде коммутатора

$$
\left[K_{12}, H_{1}-\frac{1}{2} G_{1}^{2}+H_{2}-\frac{1}{2} G_{2}^{2}\right]=0
$$

и, как в случае выражения (2.3), приходим к соотношениям

$$
H=h+\frac{1}{2} \bar{G}^{2}+\bar{H}, \quad K_{12}\left(\bar{H}_{1}+\bar{H}_{2}\right)=0=\left(\bar{H}_{1}+\bar{H}_{2}\right) K_{12} \quad \Longleftrightarrow \quad \bar{H}_{a b}=-\epsilon \bar{H}_{b a} .
$$

Таким образом, $\epsilon$-симметричная часть матрицы $H$ выражается через $\epsilon$-симметричную часть матрицы $\bar{G}^{2}$ :

$$
H_{a b}+\epsilon H_{b a}=\bar{G}_{a b}^{2}+\beta \bar{G}_{a b}+2 h \varepsilon_{a b} .
$$

3.3. Симметрия матрицы $H$. Следующие два соотношения:

$$
\begin{gathered}
\mathfrak{C}^{(2,4)}=\left[G_{1}+P_{12}-\epsilon K_{12}, H_{2}\right]=0, \\
\mathfrak{C}^{(2,5)}=-P_{12} \mathfrak{C}^{(2,4)} P_{12}=\left[H_{1}, G_{2}+P_{12}-\epsilon K_{12}\right]=0,
\end{gathered}
$$

отражают симметрию матрицы $H$ или $\bar{H}$. Алгебра Ли действует на эти соотношения в присоединенном представлении. Мы можем переписать их в терминах лишь $\bar{G}$ и $\bar{H}$ :

$$
\begin{gathered}
\overline{\mathfrak{C}}^{(2,4)}=\left[\bar{G}_{1}+P_{12}-\epsilon K_{12}, \bar{H}_{2}\right]=0, \\
\overline{\mathfrak{C}}^{(2,5)}=-P_{12} \overline{\mathfrak{C}}^{(2,4)} P_{12}=\left[\bar{H}_{1}, \bar{G}_{2}+P_{12}-\epsilon K_{12}\right]=0,
\end{gathered}
$$


поскольку є-симметричные части исчезают в силу предыдущих связей. По аналогии с равенствами (3.5) получаем, что

$$
\begin{array}{ll}
\overline{\mathfrak{C}}_{L}^{(2,4)}=K_{12}\left(\left[\bar{G}_{1}, \bar{H}_{2}\right]-2 \beta \bar{H}_{2}\right), & \overline{\mathfrak{C}}_{R}^{(2,4)}=\left(\left[\bar{G}_{1}, \bar{H}_{2}\right]+2 \beta \bar{H}_{2}\right) K_{12}, \\
\overline{\mathfrak{C}}_{L}^{(2,5)}=K_{12}\left(\left[\bar{H}_{1}, \bar{G}_{2}\right]+2 \beta \bar{H}_{1}\right), & \overline{\mathfrak{C}}_{R}^{(2,5)}=\left(\left[\bar{H}_{1}, \bar{G}_{2}\right]-2 \beta \bar{H}_{1}\right) K_{12} .
\end{array}
$$

3.4. Антикоммутатор, $\epsilon$-антисимметричная часть матрицы $H$. Условие интегрируемости шестой связи можно получить посредством умножения (2.8) на $1-\epsilon P_{12}$ :

$$
\begin{aligned}
\mathfrak{C}^{(2,6)}\left(1-\epsilon P_{12}\right) & =\epsilon K_{12}\left(\left[H_{1}, G_{2}\right]+\left[G_{1}, H_{2}\right]+2 \beta\left(H_{1}-H_{2}\right)\right)= \\
& =\epsilon K_{12}\left(2\left(H_{2}-H_{1}\right) P_{12}+2 \beta\left(H_{1}-H_{2}\right)+\epsilon\left[K_{12}, H_{2}-H_{1}\right]\right)= \\
& =\epsilon K_{12}\left(2 \beta\left(H_{2}-H_{1}\right)+2 \beta\left(H_{1}-H_{2}\right)-\epsilon\left(H_{2}-H_{1}\right) K_{12}\right)=0 .
\end{aligned}
$$

Последнее слагаемое тождественно равно нулю, в то время как первые два взаимно уничтожаются. Таким образом, шестая связь интегрируема. Далее,

$$
\begin{aligned}
(1+ & \left.\epsilon P_{12}\right) \mathfrak{C}^{(2,6)}\left(1+\epsilon P_{12}\right)-2\left[K_{12}, \bar{H}_{1} \bar{G}_{2}+\bar{H}_{2} \bar{G}_{1}+\bar{G}_{1} \bar{H}_{2}+\bar{G}_{2} \bar{H}_{1}+g\left(\bar{G}_{1}^{2}+\bar{G}_{2}^{2}\right)\right]= \\
= & K_{12}\left(2 \bar{G}_{1} \bar{G}_{2}^{2}+2 \bar{G}_{1}^{2} \bar{G}_{2}-\left[\bar{G}_{1}^{2}, \bar{G}_{2}\right]-\left[\bar{G}_{1}, \bar{G}_{2}^{2}\right]\right)- \\
& \left.-\left(2 \bar{G}_{2} \bar{G}_{1}^{2}+2 \bar{G}_{2}^{2} \bar{G}_{1}+\left[\bar{G}_{1}, \bar{G}_{2}^{2}\right]+\left[\bar{G}_{1}^{2}, \bar{G}_{2}\right]\right)\right) K_{12}= \\
= & K_{12}\left[\bar{G}_{1}, \bar{G}_{2}\right]\left(2 \bar{G}_{2}-\bar{G}_{2}-\bar{G}_{1}\right)-\left(\bar{G}_{1}+\bar{G}_{2}-2 \bar{G}_{2}\right)\left[\bar{G}_{1}, \bar{G}_{2}\right] K_{12}= \\
= & 2 \beta\left(K_{12}\left(\bar{G}_{2}\left(\bar{G}_{2}-\bar{G}_{1}\right)+\left(\bar{G}_{1}-\bar{G}_{2}\right) \bar{G}_{2}\right) K_{12}\right)=2 \beta\left[K_{12}, \bar{G}_{1}^{2}+\bar{G}_{2}^{2}\right] .
\end{aligned}
$$

Отметим, что в силу упомянутой выше интегрируемости $\mathfrak{C}^{(2,6)}$ данное соотношение не налагает дополнительных ограничений.

Итак, в силу шестого условия связи (2.8)

$$
-2 \overline{\mathfrak{C}}^{(2,6)}=\left[K_{12}, \bar{H}_{1} \bar{G}_{1}+\bar{H}_{2} \bar{G}_{2}+\bar{G}_{1} \bar{H}_{1}+\bar{G}_{2} \bar{H}_{2}-(\beta+g)\left(\bar{G}_{1}^{2}+\bar{G}_{2}^{2}\right)\right]=0 .
$$

Обозначим $F=\bar{H} \bar{G}+\bar{G} \bar{H}$, тогда из формулы (3.15) естественным образом вытекает, что

$$
F=f+(g+\beta) \bar{G}^{2}+\bar{F}, \quad K_{12}\left(\bar{F}_{1}+\bar{F}_{2}\right)=0=\left(\bar{F}_{1}+\bar{F}_{2}\right) K_{12} .
$$

С другой стороны, объединяя формулы (3.12), (3.13) и используя (3.2), (3.7), записываем равенства

$$
\overline{\mathfrak{C}}_{L}^{(2,4)}+\overline{\mathfrak{C}}_{L}^{(2,5)}=K_{12}\left(\bar{H}_{1} \bar{G}_{1}-\bar{H}_{2} \bar{G}_{2}+\bar{G}_{1} \bar{H}_{1}-\bar{G}_{2} \bar{H}_{2}-2 \beta\left(\bar{H}_{1}-\bar{H}_{2}\right)\right)=0
$$

и аналогичные равенства для $\overline{\mathfrak{C}}_{R}^{(2,4)}+\overline{\mathfrak{C}}_{R}^{(2,5)}$. Они означают, что разность $F-2 \beta \bar{H}$ является $\epsilon$-симметричной и $\bar{F}-2 \beta \bar{H}=\beta(g+\beta) \bar{G}$. Окончательно для шестой связи получаем, что

$$
F-2 \beta \bar{H}=\bar{H} \bar{G}+\bar{G} \bar{H}-2 \beta \bar{H}=f+(g+\beta)\left(\bar{G}^{2}+\beta \bar{G}\right) .
$$

Таким образом, бесследовая $\epsilon$-симметричная часть антикоммутатора матрицы $\bar{H}$ с $\bar{G}$ пропорциональна бесследовой $\epsilon$-симметричной части $\bar{G}^{2}$, и $2 \beta \bar{H}$ - ее $\epsilon$-антисимметричная часть. 
3.5. Коммутатор, $\epsilon$-антисимметричная часть матрицы $H$. Рассмотрим теперь седьмую связь $\mathfrak{C}^{(2,7)}$ :

$$
\begin{aligned}
\mathfrak{C}^{(2,7)}= & {\left[H_{1}, H_{2}\right]+\left(\bar{G}_{2} H_{1}-H_{2} \bar{G}_{1}\right) P_{12}-\epsilon K_{12}\left(\bar{G}_{1}-\beta\right) H_{2}+\epsilon H_{2}\left(\bar{G}_{1}-\beta\right) K_{12}+} \\
& +g\left[P_{12}-\epsilon K_{12}, H_{2}\right]=\left[\bar{H}_{1}, \bar{H}_{2}\right]+\frac{1}{2}\left[\bar{H}_{1}, \bar{G}_{2}^{2}\right]+\frac{1}{2}\left[\bar{G}_{1}^{2}, \bar{H}_{2}\right]+ \\
& +\left(\bar{G}_{2} \bar{H}_{1}-\bar{H}_{2} \bar{G}_{1}\right) P_{12}+\frac{1}{2}\left(\bar{G}_{2} \bar{G}_{1}^{2}-\bar{G}_{2}^{2} \bar{G}_{1}\right) P_{12}-\epsilon K_{12}\left(\bar{G}_{1}-\beta\right)\left(\bar{H}_{2}+\frac{1}{2} \bar{G}_{2}^{2}\right)+ \\
& +\epsilon\left(\bar{H}_{2}+\frac{1}{2} \bar{G}_{2}^{2}\right)\left(\bar{G}_{1}-\beta\right) K_{12}+g\left[P_{12}-\epsilon K_{12},\left(\bar{H}_{2}+\frac{1}{2} \bar{G}_{2}^{2}\right)\right]+ \\
& +\frac{1}{2}\left[\bar{G}_{1}^{2}, \bar{G}_{2}^{2}\right]-h\left[P_{12}-\epsilon K_{12}, \bar{G}_{2}\right]= \\
= & {\left[\bar{H}_{1}, \bar{H}_{2}\right]-\frac{1}{4}\left[P_{12}-\epsilon K_{12}, \bar{G}_{2}^{3}+2\left\{\bar{H}_{2}, \bar{G}_{2}\right\}-4 g \bar{H}_{2}-2 g \bar{G}_{2}^{2}+4 h \bar{G}_{2}\right]+} \\
& +\frac{1}{4}\left(\bar{G}_{2} \bar{G}_{1}^{2}-\bar{G}_{2}^{2} \bar{G}_{1}\right) P_{12}+\frac{\epsilon}{4}\left(\bar{G}_{2}^{2} K_{12} \bar{G}_{2}-\bar{G}_{2} \bar{K}_{12} G_{2}^{2}\right) .
\end{aligned}
$$

Принимая во внимание равенство (3.18), мы видим, что $\bar{H}$ входит только в первые два слагаемых.

Рассмотрим теперь тензор (фигурные скобки обозначают антикоммутатор)

$$
\begin{aligned}
W^{a_{2}}{ }_{d c_{1} c_{2}} & =\left(\bar{G}^{a_{2}}{ }_{d}+\epsilon \delta_{d}^{a_{2}}\right) \bar{G}_{c_{1} c_{2}}+\left(\bar{G}^{a_{2}}{ }_{c_{2}}+\epsilon \delta_{c_{2}}^{a_{2}}\right) \bar{G}_{d c_{1}}+\left(\bar{G}^{a_{2}}{ }_{c_{1}}+\epsilon \delta_{c_{1}}^{a_{2}}\right) \bar{G}_{c_{2} d}= \\
& =\frac{1}{2}\left(\left\{\bar{G}^{a_{2}}, \bar{G}_{c_{1} c_{2}}\right\}+\left\{\bar{G}^{a_{2}}{ }_{c_{2}}, \bar{G}_{d c_{1}}\right\}+\left\{\bar{G}^{a_{2}}{ }_{{ }_{1}}, \bar{G}_{c_{2} d}\right\}\right) .
\end{aligned}
$$

Слагаемые в первой строке формулы (3.20) с использованием абстрактной записи для $W^{a_{2} a_{1}} c_{1} c_{2}=W^{a_{2}} d c_{1} c_{2} \varepsilon^{d a_{1}}$ имеют следующий вид:

$$
W_{12}=-\bar{G}_{2}\left(P_{12}-\epsilon K_{12}\right) \bar{G}_{2}-\epsilon\left(P_{12}-\epsilon K_{12}\right) \bar{G}_{2}+\left(\epsilon \bar{G}_{2}+1\right) \bar{G}_{1} .
$$

Оператор $W_{12}$ уничтожается матрицей $K_{12}$ :

$$
W_{12} K_{12}=0=K_{12} W_{12}
$$

в матричной записи это означает, что свертка $W^{a_{2} a_{1}} c_{1} c_{2}$ по любой паре индексов равна нулю. Аналогично, оператор $W_{12}$ уничтожается матрицей $P_{12}+\epsilon$ :

$$
W_{12} P_{12}=P_{12} W_{12}=-\epsilon W_{12} \text {. }
$$

Рассмотрим коммутатор

$$
\left[\bar{G}_{2}, W_{12}\right]=\left(\bar{G}_{2} \bar{G}_{1}^{2}-\bar{G}_{2}^{2} \bar{G}_{1}\right) P_{12}+\epsilon\left(\bar{G}_{2}^{2} K_{12} \bar{G}_{2}-\bar{G}_{2} K_{12} \bar{G}_{2}^{2}\right)+\left[P_{12}-\epsilon K_{12}, \epsilon \bar{G}_{2}^{2}+\bar{G}_{2}\right] \text {. }
$$

Сравнивая его с (3.19), приходим к выводу, что

$$
\begin{aligned}
\mathfrak{C}^{(2,7)}= & {\left[\bar{H}_{1}, \bar{H}_{2}\right]+g\left[P_{12}-\epsilon K_{12}, \bar{H}_{2}\right]-\frac{1}{4}\left(\bar{G}_{2} W_{12}-W_{12} \bar{G}_{2}\right)-} \\
& -\frac{1}{4}\left[P_{12}-\epsilon K_{12}, \bar{G}_{2}^{3}+(2 \beta+\epsilon) \bar{G}_{2}^{2}+(4 h+1) \bar{G}_{2}\right] .
\end{aligned}
$$


Вычислим теперь свертку

$$
\begin{aligned}
& K_{12} \bar{G}_{2} W_{12}=K_{12} \bar{G}_{2}\left(-\bar{G}_{2}\left(P_{12}-\epsilon K_{12}\right) \bar{G}_{2}-\epsilon\left(P_{12}-\epsilon K_{12}\right) \bar{G}_{2}+\left(\epsilon \bar{G}_{2}+1\right) \bar{G}_{1}\right)= \\
& \quad=K_{12}\left(\epsilon\left(-\left[\bar{G}_{1}, \bar{G}_{2}^{2}\right]+\bar{G}_{1} \bar{G}_{2}^{2}-\bar{G}_{1} \bar{G}_{2}^{2}-\left[\bar{G}_{1}, \bar{G}_{2}\right] \bar{G}_{2}\right)+m_{2} \bar{G}_{2}-\left[\bar{G}_{1}, \bar{G}_{2}\right]\right)= \\
& =-2 \epsilon K_{12}\left(\bar{G}_{2}^{3}+(2 \beta+\epsilon) \bar{G}_{2}^{2}+\epsilon\left(2 \beta-\frac{m_{2}}{2}\right) \bar{G}_{2}-\frac{m_{2}}{2}\right) \equiv-2 \epsilon K_{12} \chi_{2} .
\end{aligned}
$$

Применяя рассуждения, аналогичные проведенным выше, получаем равенство

$$
W_{12} \bar{G}_{2} K_{12}=-2 \epsilon \chi_{2} K_{12},
$$

где $\chi$ - уже встречавшийся бесследовый полином третьего порядка по $\bar{G}$ :

$$
\chi=\bar{G}^{3}+(2 \beta+\epsilon) \bar{G}^{2}+\epsilon\left(2 \beta-\frac{m_{2}}{2}\right) \bar{G}-\frac{m_{2}}{2}=\frac{1}{2}\left(\bar{G}^{3}-\varepsilon \bar{G}^{3 t}\right)-\left(2 \beta^{2}-\epsilon \beta+\frac{m_{2}}{2}\right) \bar{G} .
$$

Его можно переписать в терминах $\varepsilon$-антисимметричной части матрицы $\bar{G}^{3}$. В результате получаем, что седьмая связь (2.9) принимает вид

$$
\begin{aligned}
\mathfrak{C}^{(2,7)}= & {\left[\bar{H}_{1}+g\left(P_{12}-\epsilon K_{12}\right), \bar{H}_{2}\right]-\frac{1}{4}\left[G_{2}, W_{12}\right]-\frac{1}{4}\left[P_{12}-\epsilon K_{12}, \chi_{2}+\alpha \bar{G}_{2}\right]=} \\
= & {\left[\bar{H}_{1}+g\left(P_{12}-\epsilon K_{12}\right), \bar{H}_{2}\right]-\frac{1}{4}\left[\bar{G}_{2}, W_{12}\right]+\frac{\alpha}{4}\left[\bar{G}_{1}, \bar{G}_{2}\right]-} \\
& -\frac{1}{8} K_{12}\left[\bar{G}_{2}, W_{12}\right]-\frac{1}{8}\left[\bar{G}_{2}, W_{12}\right] K_{12}-\frac{1}{4}\left[P_{12}, \chi_{2}\right] .
\end{aligned}
$$

Наконец, последняя восьмая связь (2.10) сводится к линейной комбинации предыдущих выражений:

$$
\mathfrak{C}^{(2,8)}\left(1-\epsilon P_{12}\right)=\epsilon K_{12} \mathfrak{C}^{(2,7)}+\frac{1}{2} \mathfrak{C}^{(2,6)}\left(K_{12}-\epsilon n\right) .
$$

Таким образом, шестая и седьмая связи приводят к следующим условиям для $\varepsilon$-антисимметричной части $H$ : антикоммутатор $H$ с $\bar{G}$ и коммутаторы их элементов соответствуют выражениям, записанным в терминах $G$. В этих выражениях присутствует кубический полином по $\bar{G}$. Сравнивая с решением первого порядка, когда бесследовая $\epsilon$-симметричная часть матрицы $\bar{G}^{2}$ обращается в ноль, здесь мы видим, что именно данный член определяет $\epsilon$-симметричную часть матрицы $H$ и антикоммутатор є-антисимметричной части матрицы $H$. Более того, коммутаторы элементов матрицы $\bar{H}$ приводят к ограничениям на $\epsilon$-антисимметричную часть матрицы $\bar{G}^{3}$.

Благодарности. Авторы признательны А. П. Исаеву за полезные обсуждения.

\section{Список литературы}

[1] Е.К. Склянин, Л.А. Тахтаджян, Л. Д. Фаддеев, "Квантовый метод обратной задачи. I", ТМФ, 40:2 (1979), 194-220.

[2] В. О. Тарасов, Л. А. Тахтаджян, Л. Д. Фаддеев, “Локальные гамильтонианы для интегрируемых квантовых моделей на решетке", ТМФ, 57:2 (1983), 163-181. 
[3] P. P. Kulish, E. K. Sklyanin, "Quantum spectral transform method. Recent developments", Integrable Quantum Field Theories (Tvärminne, Finland, 23-27 March, 1981), Lecture Notes in Physics, 151, eds. J. Hietarinta, C. Montonen, Springer, Berlin, Heidelberg, 1982, 61-119.

[4] L. D. Faddeev, "How the algebraic Bethe ansatz works for integrable models", Quantum Symmetries/Symétries Quantiques, Lectures given at the Les Houches Summer School on Theoretical Physics, Session LXIV (Les Houches, France, 1 August -8 September, 1995), eds. A. Connes, K. Kawedzki, J. Zinn-Justin, North-Holland, Amsterdam, 1998, 149-211, arXiv: hep-th/9605187.

[5] В. Г. Дринфельд, "Алгебры Хопфа и квантовое уравнение Янга-Бакстера", Докл. АН СССР. Сер. физ., 283:5 (1985), 1060-1064.

[6] В.Г. Дринфельд, "Квантовые группы”, Дифференииальная геометрия, группь Ли и механика. VIII, Зап. научн. сем. ЛОМИ, 155, Наука, Л., 1986, 18-49.

[7] A. B. Zamolodchikov, Al. B. Zamolodchikov, "Factorized $S$-Matrices in two-dimensions as the exact solutions of certain relativistic quantum field models", Ann. Phys., 120:2 (1979), 253-291; Ал. Б. Замолодчиков, Факторизованное рассеяние в асимптотически-свободных двумерных моделях квантовой теории поля, Дис. ... доктора физ.-матем. наук, ОИЯИ, Дубна, 1979.

[8] B. Berg, M. Karowski, P. Weisz, V. Kurak, "Factorized $U(n)$ symmetric $S$ matrices in twodimensions", Nucl. Phys. B, 134:1 (1978), 125-132.

[9] Н. Ю. Решетихин, "Интегрируемые модели квантовых одномерных магнетиков с $O(n)$ и $S p(2 k)$-симметрией", ТМФ, 63:3 (1985), 347-366.

[10] A.P. Isaev, Quantum groups and Yang-Baxter equations, preprint 2004-132, Max-PlankInstitut für Mathematik, Bonn, 2004, http://webdoc.sub.gwdg.de/ebook/serien/e/mpi_mathematik/2004/132.pdf.

[11] Н. Ю. Решетихин, Л. А. Тахтаджян, Л. Д. Фаддеев, “Квантование групп Ли и алгебр Ли", Алгебра и анализ, 1:1 (1989), 178-206.

[12] R. Shankar, E. Witten, "The $S$ matrix of the kinks of the $(g y \psi)^{2}$ model", Nucl. Phys. B, 141:4 (1978), 349-363; Erratum, 148:3-4 (1979), 538-539.

[13] D. Chicherin, S. Derkachov, A.P. Isaev, "Conformal group: $R$-matrix and star-triangle relation", JHEP, 04 (2013), 020, 49 pp., arXiv: 1206.4150; "The spinorial $R$-matrix", J. Phys. A: Math. Theor., 46:48 (2013), 485201, 21 pp., arXiv: 1303.4929.

[14] A. P. Isaev, D. Karakhanyan, R. Kirschner, "Orthogonal and symplectic Yangians and Yang-Baxter R operators", Nucl. Phys. B, 904 (2016), 124-147, arXiv: 1511.06152.

[15] D. Karakhanyan, R. Kirschner, "Yang-Baxter relations with orthogonal or symplectic symmetry", J. Phys. Conf. Ser., 670:1 (2016), 012029, 7 pp. 